\title{
Immobilized Particles in Gel Matrix-Type Porous Media. Homogeneous Porous Media Model
}

\author{
Manuel Mota,* J osé A. Teixeira, and Alexander Yelshin
}

Centro de Engenharia Biológica-IBQF, University of Minho, Campus de Gualtar 4710-057 Braga, Portugal

\begin{abstract}
Diffusion in pure gels and gels with immobilized cells was analyzed. A model of diffusion assuming a homogeneous cell distribution in gel was improved by introducing a tortuosity value. By theor etical analysis and numerical modeling it was shown that the tortuosity of a gel with immobilized cells is the product of two factors: (1) tortuosity generated by the cells, $T_{c}$, and (2) tortuosity of the gel matrix, $T_{g}$, both variables being a function of cell volume fraction, $\phi_{c}$. Total tortuosity is thus $T_{\Sigma}=T_{c} T_{g}$. On the basis of this approach, it was possible to analyze diffusivity data for gels with immobilized cells. It was shown that, in these systems, the diffusivity $\eta=D_{e} / D_{0}$ is a complex function of (1) diffusivity in the gel, $\eta_{g}$, and (2) diffusivity in immobilized cells, $\eta_{c}$. The developed model allowed for the description of the dependence of $D_{e} / D_{0}$ on $\phi_{c}$. Comparison with numerous published experimental data showed a good fit. Observed deviations might be explained by nonhomogeneous cell distributions inside the gel matrix.
\end{abstract}

\section{Introduction}

One of the main problems in industrial technology is the use of operations where mass transfer limitations may be the limiting step. This is the case in immobilized cell systems and in the separation of macromolecules.

Considering $D_{\mathrm{e}}$ the effective diffusion coefficient in an immobilized cells system, $D_{g}$ the effective diffusion coefficient in pure gel, and $\phi_{c}$ the volume fraction of cells in the gel, several researchers investigated the diffusion phenomena in gels and in gels with immobilized cells and described the diffusion of solutes in the gel matrix by the relation $D_{e} / D_{g} \approx\left(1-\phi_{c}\right)^{2}$. This means that tortuosity is assumed to be proportional to $1 /\left(1-\phi_{c}\right)(1-4)$. However, not all data agree with this approach. Westrin and Axelsson (1) and Hannoun and Stephanopoulos (5) pointed out the possibility of modification of gel properties due to cell immobilization. Assuming in this work that tortuosity of the gel matrix will be influenced by the presence of immobilized cells and accounting for the effect of cells in the overall tortuosity, we shall try to improve the available diffusion models for immobilized cells in gel systems.

\section{Data, Model Analysis, and Discussion}

To analyze diffusion in gels, two situations may be considered, according to the existence or absence of immobilized cells in the gel.

Diffusion in Pure Gels. The available data for the effective diffusion coefficient, $D_{g}$, of sugars in calcium al ginate gels show that the ratio $\eta_{\mathrm{g}}=\mathrm{D}_{\mathrm{g}} / \mathrm{D}_{0}$ (where $\mathrm{D}_{0}$ is the diffusion coefficient in water) varies in the range 0.67-1.0, depending on the gel concentration and on the mode of preparation $(2,5-7)$.

For diffusion of oxygen in gels (8), $D_{g}$ values obtained were $81-86 \%$ of those measured in pure water. Increasing gel concentration led to a decrease in the effective

* E-mail: mmota@deb.uminho.pt. diffusion coefficient, as previously reported (5). Gel pore size was estimated to be of the order of $150 \AA$. The observed decrease in the effective diffusion was tentatively related with the increased diffusion pathway.

The effective diffusion coefficient of bovine serum al bumin (BSA) in alginate from $L$. digitata was measured to be $1.02 \times 10^{-6}, 0.82 \times 10^{-6}$, and $0.54 \times 10^{-6} \mathrm{~cm}^{2} / \mathrm{s}$ for $\mathrm{Na}$-al ginate concentrations of $1.9 \%, 2.5 \%$, and $4 \%$, respectively. At the same time, the dependence of $D_{e}$ on the alginate source was shown (9). For glass beads (internal porosity $\epsilon=0.56$ ) filled with $1 \%$ Ca-alginate gel the glucose effective diffusion coefficient $D_{e}$ was determined as $D_{e}=2.2 \times 10^{-6} \mathrm{~cm}^{2} / \mathrm{s}$ at $30{ }^{\circ} \mathrm{C}$ and the overall tortuosity, $\mathrm{T}$, was calculated as $\mathrm{T}=1.7$ (6).

The interpretation of experimental data for gel beads may be rather complicated since gradients of gel concentration may occur inside beads. This is often related with the method of gel preparation (9). Often the gel concentration near the surface becomes higher than in the core of the gel beads and the diffusion coefficient is smaller than in homogeneous gel beads. For a $2.0 \%$ gel, a $2.9 \%$ gel concentration was measured at the surface and a value of $1.9 \%$ was obtained in the center of the beads. In the nonhomogeneous case, $D_{e}$ decreased $20-50 \%$ in comparison with diffusion in homogeneous gel beads. The analysis of the three methods used for determining the effective diffusion coefficient $D_{e}$ in different types of immobilization systems showed that the membrane and cylindrical techniques give more accurate results of $D_{e}$ than the bead method (10). Thus, for further analysis, data determined with the membrane method will be used.

Data presented for mass transfer in gels by several authors $(3,5-7,11,12)$ show that the increase in gel concentration is accompanied by a decrease in diffusivity: $\eta_{\mathrm{g}}=\mathrm{D}_{\mathrm{g}} / \mathrm{D}_{0}=\epsilon_{\mathrm{g}} / \mathrm{T}_{\mathrm{g}}$, where $\epsilon_{\mathrm{g}}$ is the gel porosity or void fraction, and $\mathrm{T}_{\mathrm{g}}$ is the gel pore network tortuosity. Moreover, the effect of porosity when $T_{g}=1.0$ is assumed is not enough to explain the decrease in diffusivity. 
To illustrate this point, an example of the diffusion in an al ginate membranetaken from the experiment (5) will be considered. According to these authors, the diffusivity of glucose and ethanol in $2 \%$ al ginate membrane was $\eta_{g}$ $=0.88$ and 0.91 , respectively, whereas in a $4 \%$ gel, $\eta_{\mathrm{g}}=$ 0.71 for glucose and $\eta_{\mathrm{g}}=0.7$ for ethanol. On the basis ofauthor's estimations for gel porosity, $\epsilon_{\mathrm{g}}$, of 0.98 and 0.96 for $2 \%$ and $4 \%$ gels, respectively, the tortuosity can be calculated by the expression $\mathrm{T}_{\mathrm{g}}=\epsilon_{\mathrm{g}} / \eta_{\mathrm{g}}$. Hence, we obtain, for a $2 \%$ al ginate gel $\mathrm{T}_{\mathrm{g}}=1.08-1.11$ and for $4 \%$ gel $\mathrm{T}_{\mathrm{g}}=$ 1.352-1.37. Therefore, the increase in tortuosity with gel concentration is much higher than the decrease in porosity.

The differences found in this example may be due to the fact that, with a growing polymer concentration in the gel, a more tortuous pathway for the diffusing species may be expected, or in other words, the structural changes induced in the gel matrix by the addition of alginate may give rise to a significant increase in tortuosity.

Diffusion in Gels with Immobilized Cells. For diffusion in gels with immobilized cells, a large amount of data available in bibliography can be used for data analysis $(2,4,10,13)$. Gels with immobilized inactivated and activated cells may be analyzed in different ways (13). A gel with immobilized inactivated cells can be considered as a system with homogeneous cell distribution. When cell growth occurs in the matrix, cells may concentrate near the gel matrix surface and/or form microcolonies. This system should be treated as a porous medium with nonhomogeneous cell distribution or/and as bidisperse materials. Microcolonies may be considered as microporous particles (2).

Depending on the type of microorganisms, the following situations can be expected: (1) Cells with small size distribution can be simulated as a monosized system (2). (2) Cells with a wide range of size distribution (mixture of large, moderate, and small particles) may form dense porous media with a porosity smaller than 0.4 (see, for example, ref 14).

Equations used to explain diffusion phenomena in gels with immobilized cells can be classified in two types:

1. E quations that include the function $D_{e} / D_{0}\left(D_{e}\right.$ is the effective diffusion coefficient for gel with immobilized cells). F or a volume fraction of immobilized cells $\phi_{\mathrm{c}}=0$, the boundary condition is $D_{\Theta} / D_{0}=D_{e} / D_{0}<1$.

2. Equations that include the function $D_{e} / D_{g}$. The models for this case present a big deviation for large cell volume fractions.

The tortuosity dependence on cell volume fraction as well as on polymer concentration is the main problem when modeling immobilized cells systems in a gel matrix. According to the diffusion theory, the dependence of $D_{e} /$ $D_{g}$ on tortuosity can be represented as follows:

$$
D_{e} / D_{g}=\epsilon_{c} / T\left(\epsilon_{c}\right)
$$

where $T\left(\epsilon_{\mathrm{c}}\right)$ is the tortuosity factor expressed as a function of porosity or cell volume fraction $\epsilon_{\mathrm{c}}=1-\phi_{\mathrm{c}}$.

Attention must be paid to the fact that for the region $\phi_{\mathrm{c}}=0-0.4$ (which is the most usual situation), we have crumbly porous media. Biological cells can be mainly represented as spherical or with a spheroid shape. For these porous media, the dependence of $T$ on $\epsilon_{\mathrm{c}}$ expressed as $1 / \epsilon_{\mathrm{c}}$ should not be expected (15).

Depending on porous media structure, the tortuosity may have other representations. Tortuosity was determined in ref 16 as $\mathrm{T}=1-0.5 \ln (\epsilon)$. F or packed beds the ratio $\eta=D_{e} / D_{0}$ was determined from the analogy with electrical conductivity (17) as $\eta=\epsilon /(\epsilon+\mathrm{k}(1-\epsilon))$, where for spherical particles $k=1.5$ (shape factor). In turn, using $\mathrm{D}_{\mathrm{e}}=\epsilon \mathrm{D}_{0} / \mathrm{T}$, (18) the tortuosity was calculated with the expression $\mathrm{T}=1.5-\epsilon / 2$ (18).

\section{Proposed Model for Tortuosity Analysis}

Diffusion in Pure Gels. As was mentioned above, gel properties are sensitive to polymer concentration. Therefore the effective diffusion coefficient in gel $D_{g}$ should be estimated through the expression used in mass transfer processes:

$$
\mathrm{D}_{\mathrm{g}}=\mathrm{D}_{0} \frac{\left(1-\phi_{\mathrm{p}}\right)}{\mathrm{T}_{\mathrm{g}}}=\mathrm{D}_{0} \frac{\epsilon_{\mathrm{g}}}{\mathrm{T}_{\mathrm{g}}}
$$

where $\epsilon_{\mathrm{g}} \approx 1-\phi_{\mathrm{p}}$ is the gel void fraction (porosity), and $\mathrm{T}_{\mathrm{g}}$ is the molecule path tortuosity in a gel matrix for a defined structure and a defined diffusing molecule. In addition, tortuosity can provide some information on gel structure. On the basis of microphotographs of different gel types with and without immobilized cells, we can simulate the gel matrix as a cellular structure with permeable holed walls $(9,12,19-24)$. Examples of twodimensional graphs are shown in Figure 1.

To have an estimation of the tortuosity variation for different situations, let us then consider a simplified beehive compartment model representing three gel structures with slight alterations (see Figure 1). Structure a will then be a stretched gel structure, structure $b$ is a relaxed gel matrix, and structure c will represent a simplified compressed matrix.

Using this simple geometrical approach it is easy now to calculate the maximal geometrical pathway tortuosity by the ratio $\mathrm{L} / \mathrm{L}_{0}$.

Considering a hexagonal geometry, $\mathrm{L}_{0}=\mathrm{a} \sqrt{3} / 2$ and $L_{0}=k a \sqrt{3} / 2$ for configurations $a$ and $c$. The value for $k$ will be greater than the unity for all the stretched structures and lesser than 1 for the compressed structures.

Therefore, the tortuosity may be calculated as

$$
L / L_{0}=\sqrt{L_{0}+(3 a / 2)^{2}} / L_{0}=\sqrt{1+3 / k^{2}}
$$

The resulting boundary (maximal) tortuosity for different $k$ values may now be calculated: (a) $1.32(k=2)$; (b) 2.0 $(\mathrm{k}=1)$; and (c) $2.78(\mathrm{k}=0.667)$. This means that the gel tortuosity may vary by more than 2-fold, simply by stretching or compressing the gel compartments, which might explain in part the results reported by (5).

Diffusion in Gels with Immobilized Cells. In this case, tortuosity values can be estimated by adding the tortuosity related with cells embedded in the matrix space to the tortuosity increase of gel matrix described above.

Let us now consider a triangular zigzag configuration as a simplified description of a gel matrix filled with cells (Figure 2a). A molecule entering the gel free system from the left, in the presence of cells only, will move throughout a path with a tortuosity $\mathrm{T}_{\mathrm{c}}$ (large dotted triangles), which will be defined as large-scale tortuosity. Largescale tortuosity is $\mathrm{T}_{\mathrm{c}}=\mathrm{L}_{\mathrm{ed}} \mathrm{L}=1 / \cos (\psi)$ where $\mathrm{L}_{\mathrm{ec}}$ is the molecule path length due to the presence of cells, Figure $2 \mathrm{a}$, corresponding to the broken dotted line with fragments of $L^{\prime}$ length. The entire gel matrix thickness is represented by $L$. The mol ecule pathway in the pure gel matrix is represented by the solid broken line formed by 


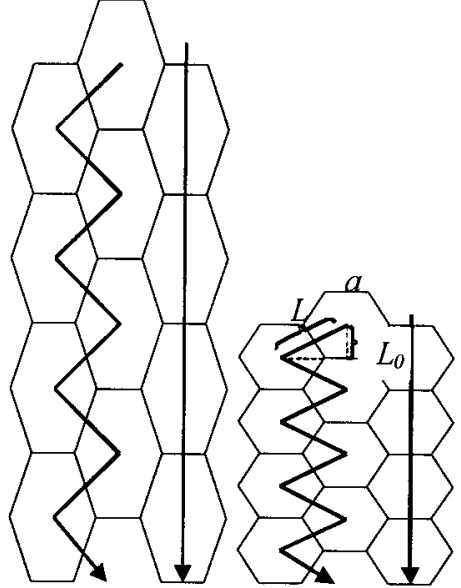

(a) (b)

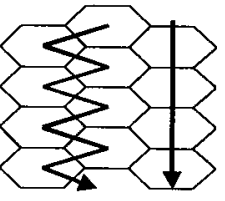

(c)
Figure 1. Simplified geometrical gel structure interpretation: (a) low polymer concentration; (b) moderate polymer concentration; (c) high polymer concentration. Straight arrows correspond to the minimal diffusion path length in the gel matrix; folded arrows represent the maximal diffusion path length in the gel matrix.

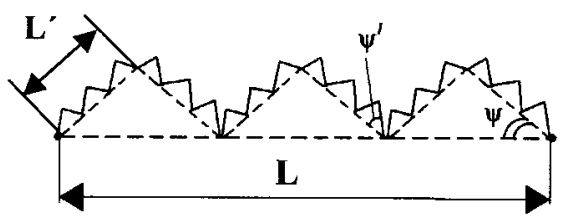

(a)

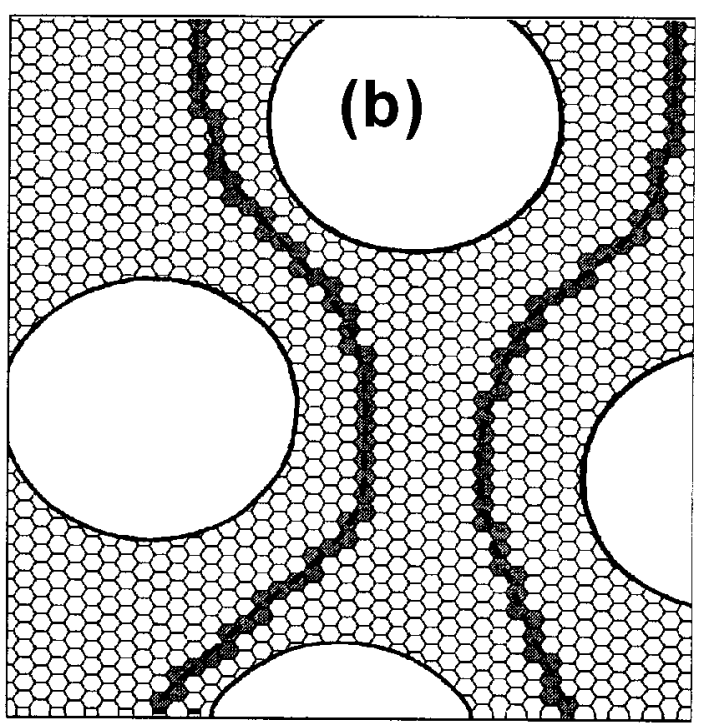

Figure 2. Schematical representations of gel structure: (a) simplified pore channel; (b) gel matrix with immobilized cells. Dotted line is the tortuosity created by cells in the gel matrix, and gray units chain is the total path in the gel matrix with cells.

the small triangles. This pathway has a tortuosity $\mathrm{T}_{\mathrm{g}}$ that we will define as small-scale tortuosity. In the latter case, the tortuosity is $T_{g}=L_{\Sigma} / L_{e c}=1 / \cos \left(\psi^{\prime}\right)$, where $L_{\Sigma}$ is the overall molecule path length in gel with immobilized cells. The total molecule path length in the channel is shown in Figure $2 \mathrm{a}$ as a broken solid line. So, the total tortuosity will be

$$
\mathrm{T}_{\Sigma}=\mathrm{L}_{\Sigma} / \mathrm{L}=\frac{\mathrm{L}_{\Sigma}}{\mathrm{L}_{\mathrm{ec}}} \cdot \frac{\mathrm{L}_{\mathrm{ec}}}{\mathrm{L}}=\mathrm{T}_{\mathrm{c}} \cdot \mathrm{L}_{\mathrm{g}}
$$

In the particular case of Figure 2a, the overall tortuosity is $\mathrm{T}_{\Sigma}=\left\{1 / \cos \left(\psi^{\prime}\right)\right\} \cdot\{1 / \cos (\psi)\}$. When assuming $\psi^{\prime}=\psi$, then $\mathrm{T}_{\Sigma}=\mathrm{T}^{2}=1 / \cos ^{2}(\psi)$.

A model of a zigzag pore channel for a gel with immobilized cells is shown in Figure $2 \mathrm{~b}$. In Figure $2 \mathrm{~b}$, two hypothetical paths in the gel matrix with immobilized cells are shown. The left pathway has a tortuosity $\mathrm{T}_{\Sigma}=1.23$, when the tortuosity created by cells is only $T_{c}=1.11$. For the right pathway, we have $T_{\Sigma}=$ 1.203 and $T_{c}=1.07$. The pathway due to the presence of cells is also influenced by the gel matrix as was discussed above: $T_{\Sigma}=T_{c} \cdot T_{g}$. A good illustration of a gel matrix structure and its transformation due to polymer concentration or to the presence of immobilized cells presence is shown on microphotographs in ref 20 . Gel matrix structure changes are most significant in the case of cell growth inside a gel, since in this case there will be additional compression effects onto the gel matrix as soon as formation of new cells occurs.

On the basis of this approach, it is possible to analyze data for cells immobilized in a gel system, where tortuosity looks too large sometimes. Assuming that the most general case of tortuosity dependence of $\mathrm{T}$ on $\mathrm{e}$ is $\mathrm{T} \approx$ $1 / \epsilon^{n}$, where $\mathrm{n}$ is an empirical constant, usually close to 0.5 , a quantitative analysis of the data will be done. By eq $4, T_{\Sigma}=T_{c} \cdot T_{g}=1 / \epsilon$, assuming that $T_{c} \approx 1 / \sqrt{\epsilon}$ and $T_{g}$ $\approx 1 / \sqrt{\epsilon}$.

The overall diffusivity $\eta=D_{e} / D_{0}$ for this system is a complex function of partial diffusivities in gel $\eta_{\mathrm{g}}=\mathrm{D}_{\mathrm{e}} / \mathrm{D}_{0}$ and in immobilized cells structure $\eta_{\mathrm{c}}=\mathrm{D}_{\mathrm{e}} / \mathrm{D}_{\mathrm{g}}$. Hence,

$$
\eta=\mathrm{D}_{\mathrm{e}} / \mathrm{D}_{0}=\eta_{\mathrm{c}}=\frac{\mathrm{D}_{\mathrm{g}}}{\mathrm{D}_{0}}=\eta_{\mathrm{c}} \eta_{\mathrm{g}}
$$

The diffusivity in a pure gel (without immobilized cells) is represented by the equation

$$
\eta_{\mathrm{g}}=\mathrm{D}_{\mathrm{g}} / \mathrm{D}_{0}=\epsilon_{\mathrm{g}} / \mathrm{T}_{\mathrm{g}}
$$

where $\epsilon_{\mathrm{g}}=1-\phi_{\mathrm{p}}$ is the gel porosity and $\mathrm{T}_{\mathrm{g}}$ is the pure gel tortuosity, as previously defined.

According to eq 3 , for the total two scale tortuosity of the gel matrix filled with cells

$$
\eta_{\mathrm{c}}=\mathrm{D}_{\mathrm{e}} / \mathrm{D}_{\mathrm{g}}=\frac{\epsilon_{\mathrm{c}}}{\mathrm{T}_{\Sigma}}=\frac{\epsilon_{\mathrm{c}}}{\mathrm{T}_{\mathrm{c}}\left(\epsilon_{\mathrm{c}}\right) \cdot \mathrm{T}_{\mathrm{g}}\left(\epsilon_{\mathrm{c}}\right)}
$$

where $T_{g}\left(\epsilon_{c}\right)$ is the tortuosity of gel matrix filled with cells (small-scale tortuosity) and $\mathrm{T}_{\mathrm{c}}\left(\epsilon_{\mathrm{c}}\right)$ is the tortuosity created in the matrix by the presence of cells (large-scale tortuosity). Replacing eqs 6 and 7 in eq 5, we obtain

$$
\eta=\eta_{\mathrm{g}} \eta_{\mathrm{c}}=\frac{\epsilon_{\mathrm{g}}}{\mathrm{T}_{\mathrm{g}}} \cdot \frac{\epsilon_{\mathrm{c}}}{\mathrm{T}_{\mathrm{c}}\left(\epsilon_{\mathrm{c}}\right) \cdot \mathrm{T}_{\mathrm{g}}\left(\epsilon_{\mathrm{c}}\right)}
$$

In the particular case where $\mathrm{T}_{\mathrm{g}}\left(\epsilon_{\mathrm{c}}\right)=\mathrm{T}_{\mathrm{c}}\left(\epsilon_{\mathrm{c}}\right)=\mathrm{T}$, eq 7 becomes

$$
\eta_{\mathrm{c}}=\frac{\epsilon_{\mathrm{c}}}{\mathrm{T}_{\mathrm{c}}\left(\epsilon_{\mathrm{c}}\right) \cdot \mathrm{T}_{\mathrm{g}}\left(\epsilon_{\mathrm{c}}\right)}=\frac{\epsilon_{\mathrm{c}}}{\mathrm{T}^{2}}
$$

and for $\mathrm{T}=\mathrm{T}_{\mathrm{g}}=\mathrm{T}_{\mathrm{c}}=1 / \epsilon^{0.5}$ 


$$
\eta_{\mathrm{c}}=\frac{\epsilon_{\mathrm{c}}}{\left(1 / \epsilon_{\mathrm{c}}^{0.5}\right)^{2}}=\epsilon_{\mathrm{c}}^{2}=\left(1-\phi_{\mathrm{c}}\right)^{2}
$$

which is in good agreement with existing models (1-4). For a pure gel, the boundary conditions of eq 7 are $\epsilon_{\mathrm{c}}=$ 1.0, $\mathrm{T}_{\mathrm{g}}\left(\epsilon_{\mathrm{c}}\right)=1.0$, and $\mathrm{T}_{\mathrm{c}}\left(\epsilon_{\mathrm{c}}\right)=1.0$ and, as a consequence, $\eta=\eta_{\mathrm{g}}=\epsilon_{\mathrm{g}} / \mathrm{T}_{\mathrm{g}}$.

Figure 3 represents the proposed model (eq 7) for the general case where the tortuosity created in the matrix by cells is $T_{c}\left(\epsilon_{c}\right)=1 / \epsilon_{c}^{\beta}=1 /\left(1-\phi_{c}\right)^{\beta}$ and the tortuosity of the gel matrix filled with cells is $\mathrm{T}_{\mathrm{g}}(\epsilon)=1 / \epsilon_{\mathrm{c}}^{\gamma}=1 /(1-$ $\left.\phi_{c}\right)^{\gamma}$. Thus

$$
\begin{gathered}
\eta=\mathrm{D}_{\mathrm{e}} / \mathrm{D}_{0}=\eta_{\mathrm{g}} \cdot\left(1-\phi_{\mathrm{c}}\right) \cdot\left(1-\phi_{\mathrm{c}}\right)^{\beta}\left(1-\phi_{\mathrm{c}}\right)^{\gamma}= \\
\eta_{\mathrm{g}}\left(1-\phi_{\mathrm{c}}\right)^{\alpha} \\
\alpha=1+\beta+\gamma
\end{gathered}
$$

where $\beta$ and $\gamma$ are values in the range $0-1.0$.

For tortuosity functions of the type $\mathrm{T}_{\mathrm{c}}\left(\epsilon_{\mathrm{c}}\right)=1 / \epsilon^{\beta}, \beta=$ 0.5 , and $T_{g}\left(\epsilon_{c}\right)=1 / \epsilon^{\gamma}$, the gel matrix tortuosity in a gel system with immobilized cells can be estimated as summarized in Table 1. Proposed model functions from Figure 3 were used. The gel matrix tortuosity (in comparison with pure gel tortuosity), in the case of immobilized cells, increases as a result of the increase of polymer concentration in the gel, meaning that $\gamma=\gamma$ $\left(\phi_{\mathrm{p}}\right)$ (Figure 4).

The proposed model gives more information about the system properties. If the cell characteristics such as shape and size distribution, the function $T_{c}$, are known, then the model can be used to calculate the tortuosity trend in the gel with immobilized cells for the ratio $D_{e} / D_{0}$ as well as for $D_{e} / D_{g}$. The model parameters lie in the region corresponding to real measured values of gel tortuosity with different polymer concentrations $(2,5-7)$ for $\mathrm{T}_{\mathrm{g}} \approx$ 1.0-1.4.

The model plots compared with experimental data are shown in Figures 5-7. The values of the power order in the model (Figure 5) are as follows: (1) $\alpha=1.9$; (2) $\alpha=$ 0.7 ; (3) $\alpha=0.5$; (4) $\alpha=1.65$; and (6) $\alpha=1.55$. Full information about data shown in Figures 6 and 7 is available in ref 1 .

It must be mentioned that the proposed model is based on the assumption of a homogeneous cell distribution within the gel matrix. Therefore some data, especially those related with cases where there is cellular growth, cannot be interpreted using this model in terms of average cell volume fraction. This problem will be addressed in a future work where $\alpha>2.2$ and $\alpha<1.8$. These ranges correspond to anisotropic nonhomogeneous porous media and need a new approach.

\section{Conclusion}

Diffusion in pure gels and gels with immobilized cells was analyzed. A model of diffusion in a gel with immobilized cells was devel oped assuming homogeneous cell distribution in gel. As compared with previous models, the proposed model shows an improvement in fitting real data, due to the introduction of a complex tortuosity value.

This proves that tortuosity is a parameter necessary for modeling gel systems with immobilized cells. By theoretical analysis and numerical modeling, it was shown that tortuosity of a gel with immobilized cells is the result of two factors: (1) tortuosity generated by cells inclusion and (2) tortuosity of the gel matrix. Both

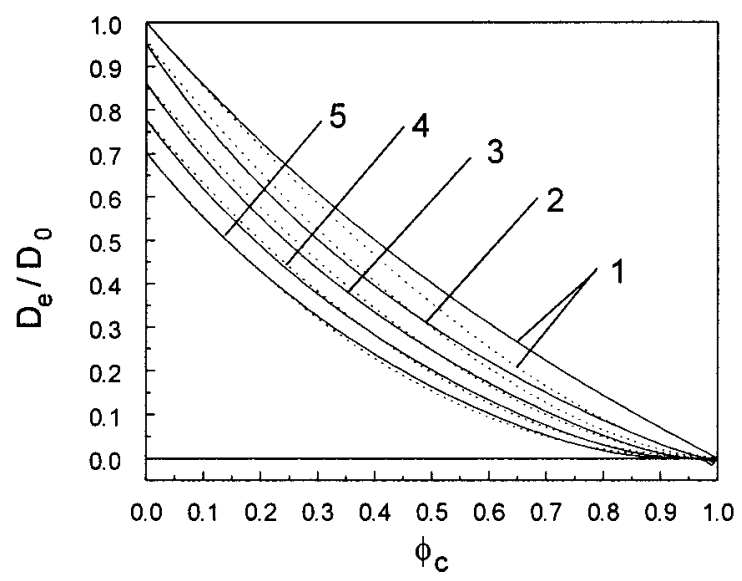

Figure 3. Dependence of $D_{e} / D_{0}$ on $\phi_{c}$ for the model of Westrin and Axelsson (1) (solid lines) and for model proposed in this work (dot lines): (1) $\phi_{\mathrm{p}}=0.0$; (2) $\phi_{\mathrm{p}}=0.01$; (3) $\phi_{\mathrm{p}}=0.03$; (4) $\phi_{\mathrm{p}}=$ 0.05 ; and (5) $\phi_{\mathrm{p}}=0.07$. Proposed model (eq 10): (1) $\mathrm{D}_{\mathrm{e}} / \mathrm{D}_{0}=(1$ $\left.-\phi_{c}\right)^{1.32} ;(2) \mathrm{D}_{\mathrm{e}} / \mathrm{D}_{0}=0.955\left(1-\phi_{\mathrm{c}}\right)^{1.68} ;(3) \mathrm{D}_{\mathrm{e}} / \mathrm{D}_{0}=0.86\left(1-\phi_{\mathrm{c}}\right)^{1.8 .}$ (4) $\mathrm{D}_{\mathrm{e}} / \mathrm{D}_{0}=0.78\left(1-\phi_{\mathrm{c}}\right)^{2}$; and $(5) \mathrm{D}_{\mathrm{e}} / \mathrm{D}_{0}=0.7\left(1-\phi_{\mathrm{c}}\right)^{2.2}$.

Table 1. Summarized Data Comparing the Model of Westrin and Axelsson (1) and Proposed Model (eqs 10 and 11) Assuming $\beta=0.5$

\begin{tabular}{cllll}
\hline gel concn, $\phi_{\mathrm{p}}$ & \multicolumn{1}{c}{$\alpha$} & \multicolumn{1}{c}{$\gamma$} & \multicolumn{1}{c}{$\eta_{\mathrm{g}}$} & \multicolumn{1}{c}{$\mathrm{T}_{\mathrm{g}}$} \\
\hline 0.0 & 1.5 & 0 & 1.0 & 1.0 \\
0.01 & 1.68 & 0.18 & 0.955 & 1.037 \\
0.03 & 1.8 & 0.3 & 0.86 & 1.128 \\
0.05 & 2.0 & 0.5 & 0.78 & 1.218 \\
0.07 & 2.2 & 0.7 & 0.7 & 1.329
\end{tabular}

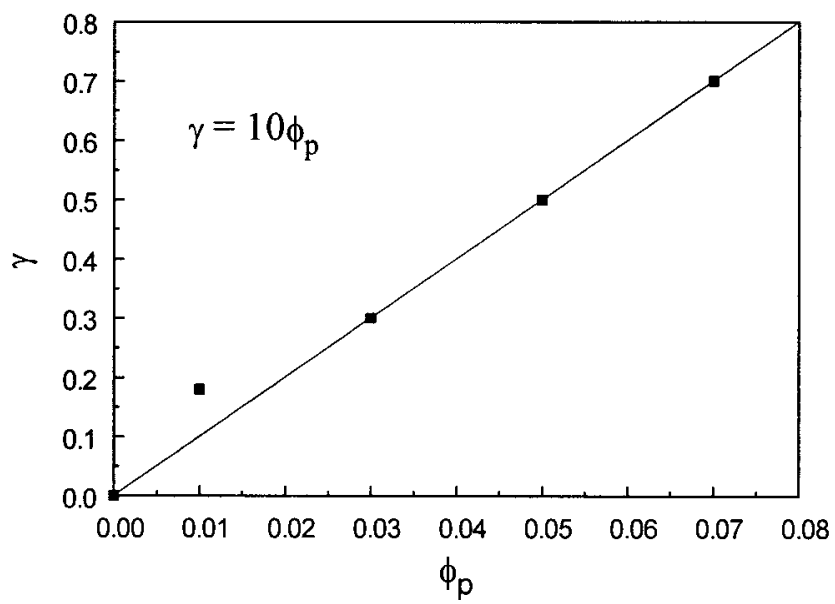

Figure 4. Dependence of $\gamma$ on the polymer concentration in gel, $\phi_{\mathrm{p}}$.

variables are a function of cell volume fraction $\phi_{\mathrm{c}}$ and can be described by an order function of $\phi_{c}$.

The developed model makes it possible to describe various types of dependence of $D_{e} / D_{0}$ on $\phi_{c}$ and also to control and optimize the diffusion inside a gel with immobilized cells. The most suitable way to reduce mass transfer resistance is to decrease the gel matrix tortuosity by controlling its structure. On the other hand, the model shows why, even with an ideal gel with tortuosity $T_{g}=$ 1.0, the presence of cells in the gel matrix will give rise to a minimal tortuosity always above 1.0.

The model was compared with numerous published experimental data, and a good approach was obtained. Nevertheless, not all experimental data can be explained on the basis of a homogeneous model, when anomalously small or large values of $D_{e} / D_{0}$ vs $\phi_{c}$ are measured. Although the present model considers the diffusivity in 


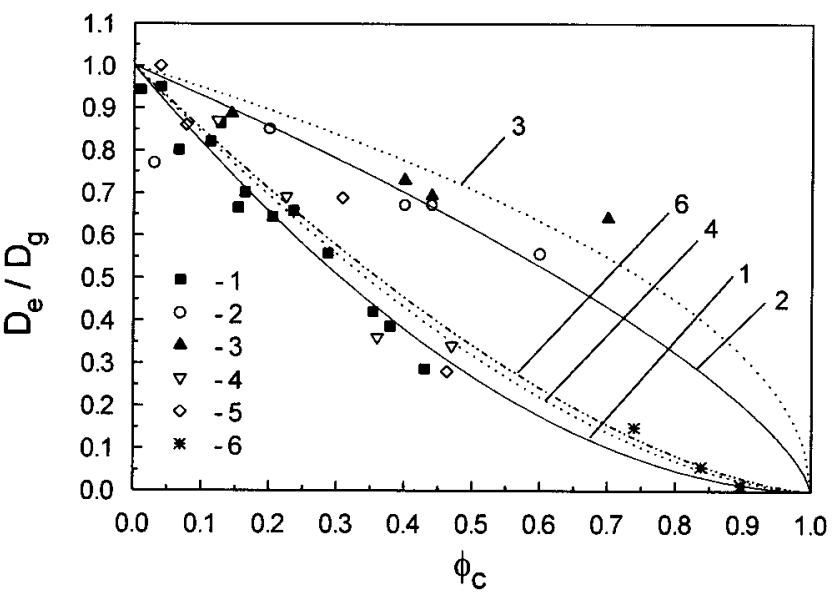

Figure 5. Experimental data and the model plots of $D_{e} / D_{g}$ vs $\phi_{c}$ : (1) $2 \%$ Ca-al ginate gel, Z. mobiliz, solute galactose (2); (2) $2 \%$ collagen and $1 \%$ agar, EAT cells, solute glucose (25); (3) Caal ginate gel $20 \mathrm{~kg} / \mathrm{m}^{3}$, Bacillus amyl ol iquefaciens, solute oxygen (8); (4) lactose (18); (5) 4\% Ca-alginate gel, Saccharomyces cerevisiae, solute glucose (13); (6) 2\% Ca-alginate gel, backer's yeast, solute acetophenone (4). Lines correspond to the model proposed in this work.

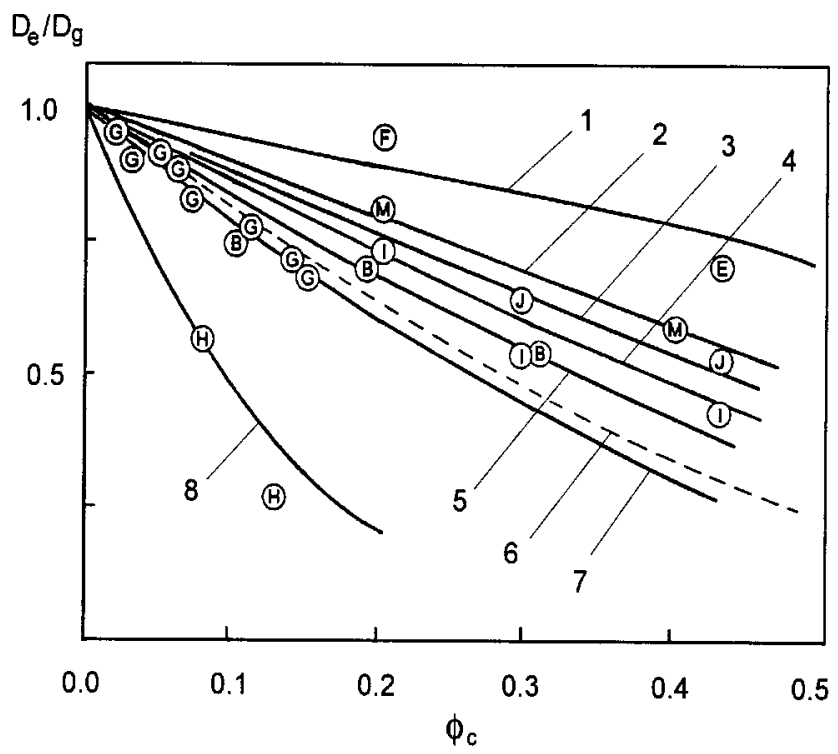

Figure 6. Comparison of experimental data from Figure 2 of ref 1 with the model proposed in this work. The values of $\alpha$ are (1) 0.45 ; (2) 0.95; (3) 1.1 ; (4) 1.3; (5) 1.6; (6) 2.0 (by eq 9); (7) 2.2; and (8) 7.0. Data have symbols as in the original work: (B) PMAAm gel + yeast; (E) Ca-alginate gel + yeast for xylose; (F) Ca-alginate gel + yeast for glucose; (G) Ca-alginate gel + bacteria; $(\mathrm{H})$ Ca-alginate gel + plant cells; (I) agar + mammalian cells for lactate; ( ) agar + mammalian cells for glucose; and (M) $\kappa$-carraginate + bacteria for oxygen.

the gel, the diffusivity in the immobilized cells, and the cell volume fraction, nonhomogeneous cell distribution is probable in several cases. Factors such as cell mean size, cell distribution inside the gel, and cell size distribution will be considered in a future work.

\section{Acknowledgment}

This work was funded by PRAXIS XXI under the grant BBC/6440/95.

\section{Notation}

$D_{c} \quad$ diffusion coefficient in the presence of cells, $\mathrm{cm}^{2} / \mathrm{s}$

$D_{e} \quad$ effective diffusion coefficient in gel with immobilized cells, $\mathrm{cm}^{2} / \mathrm{s}$

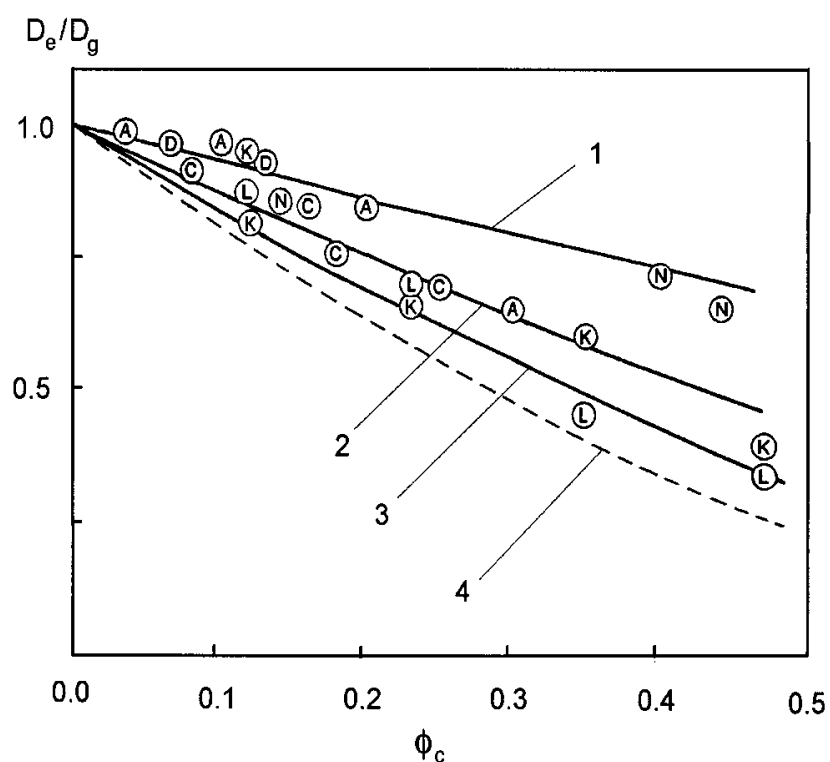

Figure 7. Comparison of experimental data from Figure 3 of ref 1 and the proposed model. The value of $\alpha$ are (1) 0.5; (2) 1.15; (3) 1.5; and (4) 2.0 (by eq 9). Data have the same symbols as in the original work: (A) PAA + bacteria for glucose; (C) PAA + bacteria for ammonium fumarate; (D) $\kappa$-carraginate + bacteria for ammonium fumarate; (K) Ca-alginate gel + yeast for lactose; (L) Ca-alginate gel + yeast for ethanol; and (N) Ca-alginate gel + bacteria for oxygen.

$D_{g} \quad$ effective diffusion coefficient in pure gel, $\mathrm{cm}^{2} / \mathrm{s}$

$\mathrm{D}_{0} \quad$ diffusion coefficient in bulk liquid, $\mathrm{cm}^{2} / \mathrm{s}$

L gel matrix thickness with immobilized cells

$T$ tortuosity factor or tortuosity

$\mathrm{T}_{\mathrm{c}} \quad$ tortuosity created by cell presence

$\mathrm{T}_{\mathrm{g}} \quad$ tortuosity of gel matrix

$\epsilon_{\mathrm{c}} \quad$ void fraction of cells in matrix

$\epsilon_{\mathrm{g}} \quad$ gel matrix porosity, $\epsilon_{\mathrm{g}}=1-\phi_{\mathrm{p}}$

$\phi_{c} \quad$ cell volume fraction for homogeneous cells distribution in the gel matrix

$\phi_{\mathrm{p}} \quad$ polymer volume fraction of gel

$\eta \quad=\mathrm{D}_{\mathrm{e}} / \mathrm{D}_{0}$, overall diffusivity

$\eta_{\mathrm{g}} \quad=\mathrm{D}_{\mathrm{g}} / \mathrm{D}_{0}$

$\eta_{\mathrm{c}} \quad=\mathrm{D}_{\mathrm{e}} / \mathrm{D}_{\mathrm{g}}$

$\alpha \quad$ see eq 10

$\beta$ and $\gamma$ order values in the range $0-1.0$, eq 9

\section{References and Notes}

(1) Westrin, B. A.; Axelsson, A. Diffusion in gels containing immobilized cells: A critical review. Biotechnol. Bioeng. 1991, 38, 439-446.

(2) Korgel, B. A.; Rotem, A.; Monbouquette, H. G. Effective diffusivity of galactose in calcium alginate gels containing immobilized Zymomonas mobilis. Biotechnol. Prog. 1992, 8, 111-117.

(3) Teixeira, J .; Mota, M.; Venancio, A. Model identification and diffusion coefficient determination of glucose and malic acid in calcium alginate membranes. Chem. Eng. J . 1994, 56, B9B14.

(4) Wu W.; Sidhoum, M.; DeLancey, G. B. Diffusion of acetophenone and phenethyl alcohol in the calcium-alginatebakers' yeast-hexane system. Bi otechnol. Bi oeng. 1994, 44, 1217-1227.

(5) Hannoun, B. J . M.; Stephanopoulos, G. Diffusion coefficient of glucose and ethanol in cell-free and cell-occupied calcium al ginate membranes. Biotechnol. Bioeng. 1986, 28, 829-835.

(6) De Backer, L.; Baron, G. Effective diffusion and tortuosity in porous-glass immobilization matrix. Appl. Microbiol. Biotechnol. 1993, 39, 281-284. 
(7) Venâncio, A.; Teixeira, J. A. Characterization of sugar diffusion coefficients in alginate mambranes. Biotechnol. Tech. 1997, 11, 183-185.

(8) Sun, Y.; Furusaki, S.; Yamauchi, A.; Ichimura, K. Diffusivity of oxygen into carriers entrapping whole cells. Biotechnol. Bioeng. 1989, 34, 55-58.

(9) Martinsen, A.; Storrø, I.; Skjåk-Bræk, G. Alginate as immobilization material: III. Diffusional properties. Bi otechnol. Bioeng. 1992, 39, 186-194.

(10) I tamunoala, G. F. Limitations of methods of determining effective diffusion coefficients in cell immobilization matrices. Biotechnol. Bioeng. 1988, 31, 714-717.

(11) Axelsson, A.; Sisak, C.; Westrin, B.;. Szajani, B. Diffusional characteristics of a swelling gel and its consequences for bioreactor performance. Chem. Eng. J . 1994, 56, B35 - B39.

(12) Fukushima, Y.; Okamura, K.: Imai, K.; Motai, H. A new immobilization technique of whole cells and enzymes with colloidal silica and alginate. Biotechnol. Bioeng. 1988, 32, 584-594.

(13) De Backer, L.; Devleminck, S.; Willaert, R.; Baron, G. Reaction and diffusion in a gel membrane reactor containing immobilized cells. Biotechnol. Bioeng. 1992, 40, 322-328.

(14) Reetz, M. T.; Zonta, A.; Simpelkamp, J. Efficient immobilization of lipases by entrapment in hydrophobic solgel materials. Biotechnol. Bioeng. 1996, 49, 527-534.

(15) Bear, J . Dynamics of Fluids in Porous Media. Elsevier: New York, 1972.

(16) Ho, Fan-Gan; Strieder, W. A variational calculation of the effective surface diffusion coefficient and tortuosity. Chem. Eng. Sci. 1981, 36, 253-258.

(17) Suzuki, M. Adsorption Engineering. Elsevier: New York 1990.

(18) Riley, M. R.; Muzzio, F. J .; Buettner, H. M.; Reyes, S. C. A simple correlation for predicting effective diffusivities in immobilized cell systems. Biotechnol. Bioeng. 1996, 49, 223227.
(19) Dean, R. C.; Karkare, S. B.; Ray, N. G.; Venkatasubramanian, K. Large scale culture of hybridoma and mammalian cells in fluidized bed bioreactors. In Bioreactor Immobilized Enzymes and Cells Fundamentals and Applications; MooYoung, M., Ed.; Elsevier: U.K., 1988; pp 132-133.

(20) Tamada, M.; Kagai, N.; Kaetsu, I. Effect of structure of polymer support on the growth of Sporotrichum cellulophilum immobilized by polymerization induced by gamma rays. Biotechnol. Bioeng. 1988, 32, 386-390.

(21) Barbotin, J. N. et al. In Physiology of I mmobilized Cells. Proceedings of International Symposium, Wageningen 1013 Dec. 1989; De Bont, J. A. M. et al., Eds.; Elsevier Science Publishers: Amsterdam, 1990; pp 487-497.

(22) Donova, M. V.; Gulevskaya, S. A.; Koshcheyenko, K. A. In Physiol ogy of I mmobilized Cells. Proceedings of I nternational Symposium, Wageningen 10-13 Dec. 1989; De Bont, J. A M. et al., Eds.; Elsevier Science Publishers: Amsterdam, 1990; pp 517-522.

(23) Guyot, J . B.; Tamponnet, C.; L ievremont, M. In Physiol ogy of I mmobilized Cells. Proceedings of I nternational Symposium, Wageningen 10-13 Dec. 1989; De Bont, J . A. M. et al., Eds.; Elsevier Science Publishers: Amsterdam, 1990; pp 243248.

(24) Ito, Y. et al. Serum-free cell culture on insulin-immobilized porous collagen beads. Biotechnol. Bioeng. 1995, 45, 144148.

(25) Chresand, T. J .; Dale, B. E.; Hanson, S. L.; Gillies, R. J . A stirred bath technique for diffusivity measurements in cell matrixes. Biotechnol. Bioeng. 1988, 32, 1029-1036.

Accepted for publication May 23, 2001.

BP010064T 